\title{
Got Beer? A Systematic Review of Beer and Exercise
}

\author{
Jaison L. Wynne and Patrick B. Wilson \\ Old Dominion University
}

\begin{abstract}
Beer is used to socialize postexercise, celebrate sport victory, and commiserate postdefeat. Rich in polyphenols, beer has antioxidant effects when consumed in moderation, but its alcohol content may confer some negative effects. Despite beer's popularity, no review has explored its effects on exercise performance, recovery, and adaptation. Thus, a systematic literature search of three databases (PubMed, SPORTDiscus, and Web of Science) was conducted by two reviewers. The search resulted in 16 studies that were appraised and reviewed. The mean PEDro score was 5.1. When individuals are looking to rehydrate postexercise, a low-alcohol beer $(<4 \%)$ may be more effective. If choosing a beer higher in alcoholic content $(>4 \%)$, it is advised to pair this with a nonalcoholic option to limit diuresis, particularly when relatively large volumes of fluid ( $>700 \mathrm{ml})$ are consumed. Adding $\mathrm{Na}^{+}$to alcoholic beer may improve rehydration by decreasing fluid losses, but palatability may decrease. These conclusions are largely based on studies that standardized beverage volume, and the results may not apply equally to situations where people ingest fluids and food ad libitum. Ingesting nonalcoholic, polyphenol-rich beer could be an effective strategy for preventing respiratory infections during heavy training. If consumed in moderation, body composition and strength qualities seem largely unaffected by beer. Mixed results that limit sweeping conclusions are owed to variations in study design (i.e., hydration and exercise protocols). Future research should incorporate exercise protocols with higher ecological validity, recruit more women, prioritize chronic study designs, and use ad libitum fluid replacement protocols for more robust conclusions.
\end{abstract}

Keywords: alcohol, dehydration, performance, recovery, training

Beer is a yeast-fermented alcoholic beverage consisting primarily of water, malted cereals, and hops (Young, n.d.). Although its alcohol content can vary from $<1 \%$ to over $15 \%$, the typical beer contains about $5 \%$ alcohol by volume (Logan et al., 1999). In 2019, $65 \%$ of Americans drank alcohol, with beer topping the poll (Gallup, 2019). Beer consumption is also highly prevalent among sportspeople. At the collegiate level, athletes are more likely than nonathletes to drink beer (Overman \& Terry, 1991) and binge drink (Nelson \& Wechsler, 2001). Furthermore, nearly four in five female athletes of a Division I sample consumed alcohol, with light beer being the most popular beverage (Martin, 1998). In elite sports, former Finnish team athletes consumed more beer per month than controls (Kontro et al., 2017). Moreover, links between alcohol advertising and sport reenforce the strong connection beer has with athletes. For National Collegiate Athletic Association basketball tournament games from 1999 to 2008, all but five of 294 nationally televised alcohol advertisements were sponsored by beer or other malt liquor producers (Babor et al., 2013), and in 2012, beer ads made up $68.1 \%$ of all alcohol-related advertisements on sport TV programming in five major metropolitan centers of Australia (O'Brien et al., 2015).

There are various mechanisms by which beer could either positively or negatively impact acute and chronic exercise-related outcomes. Beyond its obvious detrimental effects on neuromotor function, high doses of alcohol impair muscle protein synthesis (MPS; Parr et al., 2014) and stimulate diuresis (Jones, 1990), which could lead to suboptimal training adaptions and rehydration, respectively. Then again, ingesting beer can acutely increase

The authors are with the Human Performance Laboratory, Human Movement Sciences Department, Old Dominion University, Norfolk, Virginia, USA. Wilson (pbwilson@odu.edu) is corresponding author. plasma antioxidant capacity, largely due to the absorption of phenolic acids (Ghiselli et al., 2000). However, beer's total antioxidant activity and polyphenol content depend chiefly upon the style of beer and its ethanol content (Piazzon et al., 2010). Prior research on polyphenols and exercise outcomes is mixed, but some data indicate they can positively impact performance (Somerville et al., 2017) and recovery (Myburgh, 2014). This suggests that, when consumed in moderation, beer could have some positive effects for people undertaking regular training.

Several reviews on alcohol and physical activity have been published previously (Barnes, 2014; Leasure et al., 2015; PiazzaGardner \& Barry, 2012). However, the broad scope of such reviews on multiple alcoholic beverages brings with it a limited focus, making it difficult to generalize about any particular beverage. Due to variations in alcohol content, phytochemistry, and nutrient profile, there are likely to be differences between alcoholic beverages regarding their effects on exercise-related outcomes. The present systematic review centered on beer's effects on exercise performance, adaptation, and recovery.

\section{Methods}

\section{Overview}

This systematic review was carried out to identify peer-reviewed experimental research that examined the effects of beer on exercise-related outcomes (performance, adaptation, or recovery). Studies of both an acute and chronic nature were of interest. Here, we define acute studies as ones in which beer ingestion and exercise occurred within $24 \mathrm{hr}$ of each other. Chronic studies involved beer administration (or reduction in intake among regular consumers) for 1 week or longer. Furthermore, chronic studies also needed to have a regular exercise training (aerobic, resistance, 
interval, etc.) component to be included. Below are additional eligibility criteria:

(a) Types of studies: Randomized and nonrandomized human experiments.

(b) Participants: Individuals of legal drinking age able to complete exercise.

(c) Interventions: Administration of alcoholic or nonalcoholic beer acutely or chronically in conjunction with either acute exercise or chronic training. Studies that involved reducing habitual beer intake were also considered.

(d) Outcomes: Variables related to exercise performance, adaptation, or recovery were considered. Expressly, anthropometrics; body composition; MPS; muscle soreness; muscle damage; hydration status; time-trial performance; time to exhaustion; clinical outcomes (e.g., respiratory tract infections); physiological biomarkers (e.g., oxygen consumption, blood pressure, lipids, etc.); perceptual responses (e.g., exertion); and measures of muscular strength, power, or endurance were considered.

Review articles and those not available in English were excluded. Also, studies failing to clearly report beer dosage or timing of ingestion were excluded, as were theses, dissertations, conference abstracts, and articles reporting duplicate data.

\section{Search Strategy and Data Extraction}

Two reviewers independently searched three databases (PubMed, SPORTDiscus, and Web of Science) in October 2020. The search terms and strategy are listed in Table 1. The articles were screened first through title and abstract. If it remained unclear as to whether an article met the inclusionary criteria, the full text was read. After each reviewer completed their own search, they met to finalize the selected articles. The selected articles were evaluated in their entirety with applicable information extracted, including sample sizes, subject characteristics, research designs, independent variables (i.e., treatment and control conditions), and outcomes.

\section{Study Quality}

Randomized interventions were evaluated using the 11-item PEDro Scale (de Morton, 2009). Ten items are graded as 1 or 0 and totaled for a sum score. Previous studies have exhibited variability in the cutoffs for determining study quality (de Morton, 2009; Moseley et al., 2011). This review's classification system is listed in Table 2.

\section{Synthesis/Presentation of Results}

Studies were grouped based on acute or chronic design, given that beer's effects are likely to vary depending on whether it is ingested in one sitting or chronically (i.e., $>1$ week). For acute studies, outcomes were subgrouped based on categories (hydration, neuromuscular, etc.), while for chronic studies, outcomes were reviewed for studies individually, because there were only four studies and each had somewhat different outcomes. For all studies, the percentages preceding or following "beer" were used as shorthand to describe the alcohol content (e.g., $4 \%$ beer means beer that is $4 \%$ alcohol by volume).

\section{Results}

Initially, 22 articles appeared to be eligible. However, based on further discussion between the reviewers, six additional articles were excluded, with citations and reasons given in Supplemental Table 1 (available online). The search/selection process is shown in Figure 1. Fifteen of 16 included studies were scorable on PEDro (Figure 2). The mean score was 5.1, and three of 15 studies scored $\geq 6$ (Figure 3 ).

\section{Acute Studies-Samples}

Twelve of 16 studies were acute in nature (Table 3). The groups recruited included professional soccer players (Castro-Sepulveda et al., 2016), recreational athletes (Desbrow et al., 2013; Desbrow et al., 2015), college students (Desbrow et al., 2019; Flores-Salamanca \& Aragón-Vargas, 2014; Kruisselbrink et al., 2006), physically active males (Hobson \& Maughan, 2010; Jiménez-Pavón et al., 2015; Rodrigues et al., 2019; Shirreffs \& Maughan, 1997; Wijnen et al., 2016), and healthy males (Ka et al., 2003). Only two studies included females (Desbrow et al., 2019; Kruisselbrink et al., 2006); $13.5 \%$ of the participants were female. The mean ages ranged from $19.1 \pm 0.4$ to $36 \pm 9$ years. The sample sizes varied from six (Ka et al., 2003; Shirreffs \& Maughan, 1997) to 54 (Desbrow et al., 2019).

\section{Acute Studies-Exercise Tasks}

For this section, "exercise task" refers to either experimental procedures (e.g., dehydrating exercise) or exercise-related outcomes (e.g., strength/balance testing). Seven studies used moderate to vigorous exercise to dehydrate participants to a target body mass loss before beverage administration (Desbrow et al., 2013, 2015; Flores-Salamanca \& Aragón-Vargas, 2014; Hobson \& Maughan,

\section{Table 2 PEDro Scale Quality Classification}

\begin{tabular}{lcccr}
\hline Quality & Low & Fair & Moderate & High \\
\hline Sum score & $0-3$ & $4-5^{*}$ & $6-7$ & $8-10$ \\
\hline $\begin{array}{l}\text { Note. Average PEDro sum score } \\
\text { (Moseley et al., 2011). }\end{array}$ \\
*PEDro sum score mean: 5.1 (PEDro, 2020).
\end{tabular}

Table 1 Overview of the Search Strategy

\begin{tabular}{ll}
\hline Variables & Search terms \\
\hline $\begin{array}{l}\text { Group 1: Study } \\
\text { design }\end{array}$ & $\begin{array}{l}\text { randomized OR randomised OR randomly OR allocated OR placebo OR blinded OR "clinical trial” OR experiment OR } \\
\text { controlled OR crossover OR "within subjects" OR "between subjects" OR "repeated measures" OR counterbalanc* }\end{array}$ \\
$\begin{array}{ll}\text { Group 2: Beer } \\
\text { terms }\end{array}$ & beer OR lager OR ale OR "malt liquor" OR "alcoholic beverage" OR "alcohol free" \\
Group 3: Outcomes & $\begin{array}{l}\text { exercise OR recovery OR hydration OR strength OR "physical activity" OR "muscle protein synthesis" OR “muscle soreness" } \\
\text { OR "muscle damage" OR athlet* OR "body build*" OR sport* OR running OR cycling OR swimming OR sprint OR jump }\end{array}$ \\
\hline
\end{tabular}

Note. Groups were combined with "AND” (Group 1 AND Group 2 AND Group 3). 


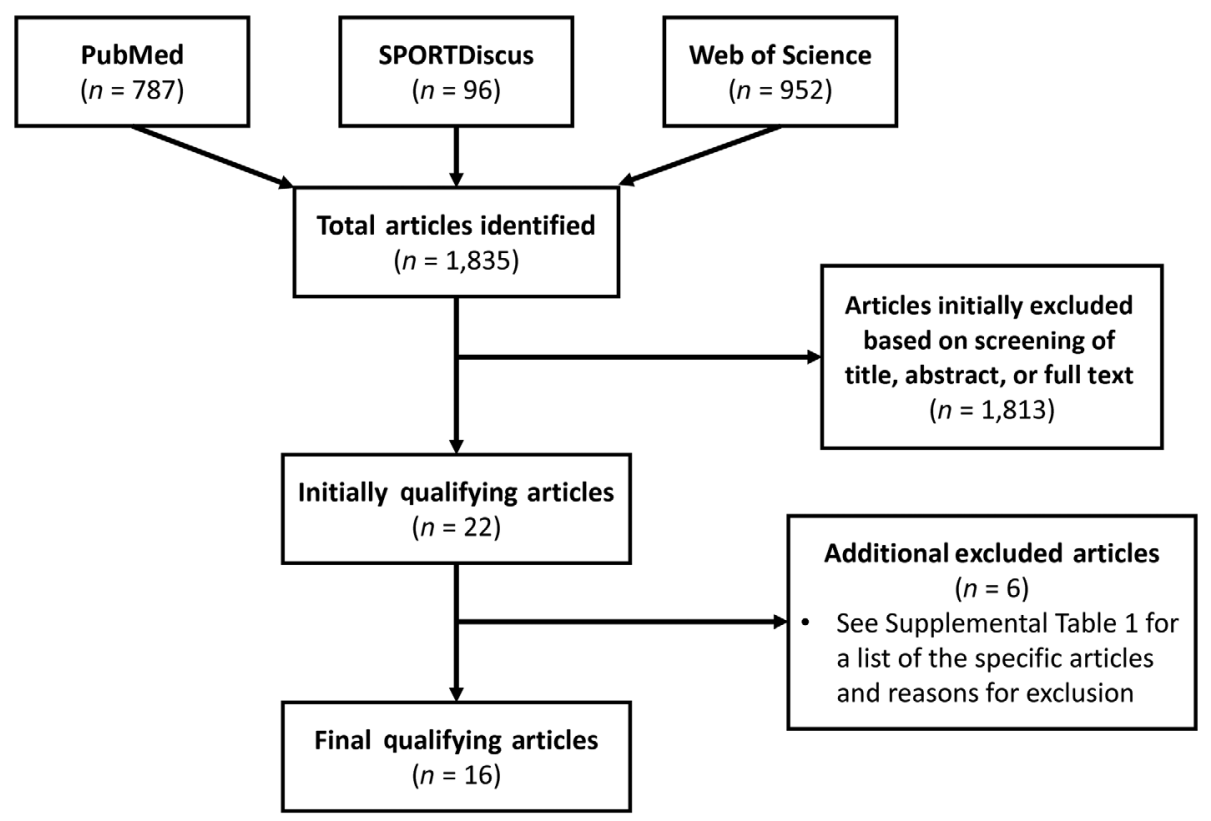

Figure 1 - An overview of the literature search and article selection process.

\begin{tabular}{|c|c|c|c|c|c|c|c|c|c|c|c|c|}
\hline Study & 1 & 2 & 3 & 4 & 5 & 6 & 7 & 8 & 9 & 10 & 11 & Score \\
\hline Castro-Sepulveda et al. (2016) & 0 & 0 & O & O & 0 & 0 & O & O & 0 & & 0 & 8 \\
\hline Cox et al. (1993) & 0 & & O & & 0 & 0 & $\bigcirc$ & O & 0 & & & 5 \\
\hline Desbrow et al. (2013) & O & & $\bigcirc$ & 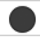 & O & 0 & $\bigcirc$ & O & O & & & 4 \\
\hline Desbrow et al. (2015) & O & & O & ? & O & O & $\bigcirc$ & O & 0 & & $\sqrt{3}$ & 4 \\
\hline Desbrow et al. (2019) & 0 & & O & 0 & 0 & $\mathrm{O}$ & $\mathrm{O}$ & $\mathrm{O}$ & $\mathrm{O}$ & 0 & & 4 \\
\hline Flores-Salamanca and Aragón-Vargas (2014) & O & & O & O & O & O & O & O & 0 & O & O & 4 \\
\hline Hobson and Maughan (2010) & O & & $\bigcirc$ & & 0 & O & O & O & $\bigcirc$ & & & 4 \\
\hline Jiménez-Pavón et al. (2015) & O & & 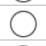 & & O & $\mathrm{O}$ & $\bigcirc$ & 0 & 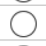 & & & 5 \\
\hline Kruisselbrink et al. (2006) & O & & O & ( & O & $\mathrm{O}$ & 0 & O & $\mathrm{O}$ & & 0 & 3 \\
\hline Molina-Hidalgo et al. (2019) & O & & O & 0 & O & O & O & O & 0 & O & O & 5 \\
\hline Molina-Hidalgo et al. (2020) & 0 & & $\bigcirc$ & $\bigcirc$ & $\bigcirc$ & 0 & 0 & 0 & 0 & O & 0 & 5 \\
\hline Rodrigues et al. (2019) & 0 & & O & O & 0 & O & 0 & O & O & O & J & 6 \\
\hline Scherr et al. (2012) & O & & 0 & O & O & O & O & O & 0 & O & O & 9 \\
\hline Shirreffs and Maughan (1997) & $\bigcirc$ & & O & O & 0 & O & $\bigcirc$ & $\bigcirc$ & O & O & & 5 \\
\hline Wijnen et al. (2016) & 0 & & 0 & & 0 & 0 & 0 & 0 & 0 & 0 & 0 & 5 \\
\hline
\end{tabular}

Figure 2 - PEDro scoring for the 15 randomized experiments. Filled circles indicate the criteria is met, while open circles indicate the criteria is not met. Item 1 is not included in the final score.

2010; Jiménez-Pavón et al., 2015; Shirreffs \& Maughan, 1997; Wijnen et al., 2016). Flores-Salamanca and Aragón-Vargas (2014) also conducted balance and reaction time outcome testing. Three studies utilized a set distance or time of aerobic exercise either before or after beverage ingestion (Castro-Sepulveda et al., 2016; Desbrow et al., 2019; Ka et al., 2003). Two studies employed either neuromuscular outcome testing alone (Rodrigues et al., 2019) or neuromuscular and aerobic outcome testing (Kruisselbrink et al., 2006).

\section{Acute Studies-Beer Consumption Procedures}

One study provided beverages $45 \mathrm{~min}$ preexercise (CastroSepulveda et al., 2016), while most used postexercise administration. Several studies divided beverage intake into equal doses throughout various time ranges (Desbrow et al., 2013, 2015; Flores-Salamanca \& Aragón-Vargas, 2014; Kruisselbrink et al., 2006; Rodrigues et al., 2019; Shirreffs \& Maughan, 1997; Wijnen et al., 2016), while two employed ad libitum drinking (Desbrow et al., 2019; Jiménez-Pavón et al., 2015).

In studies looking at postexercise consumption, the time between exercise cessation and consumption varied. One study had participants consume drinks in the morning after prior-evening exercise (Hobson \& Maughan, 2010). Most studies, however, administered beverages starting no more than 30-60 min postexercise (Desbrow et al., 2013, 2015, 2019; Flores-Salamanca \& Aragón-Vargas, 2014; Jiménez-Pavón et al., 2015; Ka et al., 2003; Shirreffs \& Maughan, 1997; Wijnen et al., 2016).

Regarding beverage volume, the procedures varied between studies. When rehydrating postexercise, five studies had participants 


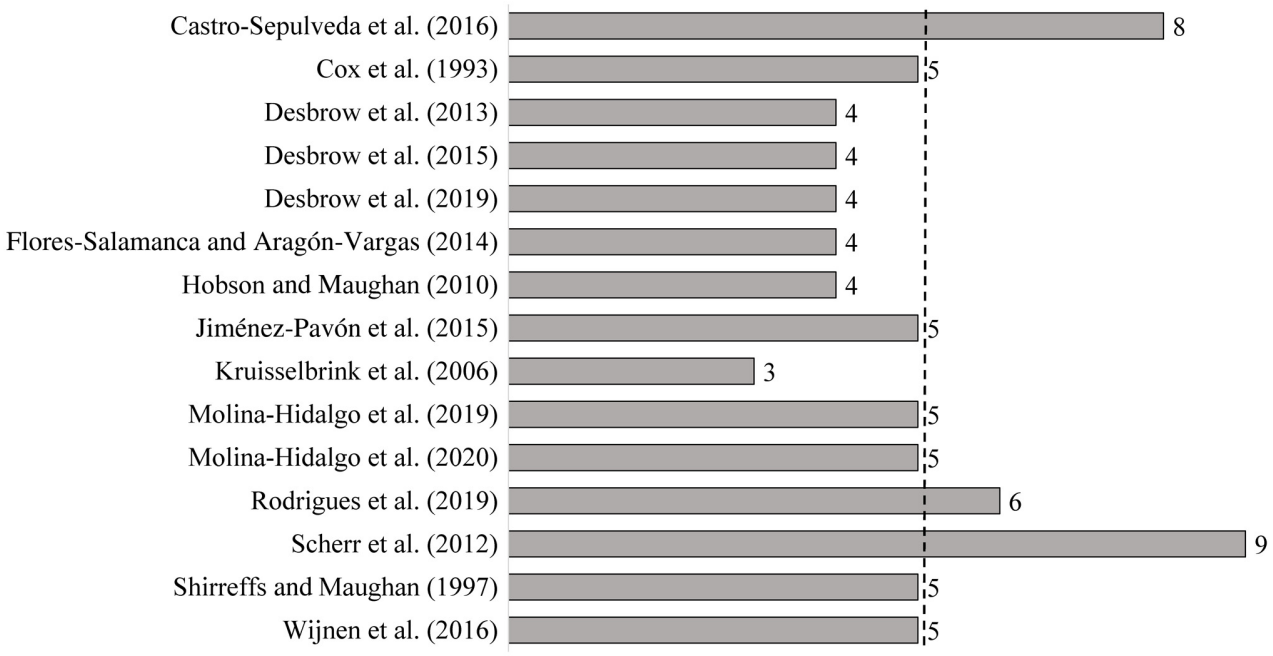

Figure 3 - Visual representation of relative study quality on the PEDro Scale. The dashed line represents the average value from the 15 scored studies.

drink to 150\% (Desbrow et al., 2013, 2015; Shirreffs \& Maughan, 1997 ) or $100 \%$ of body mass losses (Flores-Salamanca \& AragónVargas, 2014; Wijnen et al., 2016), whereas two studies utilized amounts expressed as grams or milliliters per kilogram of body mass (Ka et al., 2003; Rodrigues et al., 2019). Five studies employed drinking ad libitum (Desbrow et al., 2019; Jiménez-Pavón et al., 2015 ) or to a preset volume (Castro-Sepulveda et al., 2016; Hobson \& Maughan, 2010; Kruisselbrink et al., 2006).

\section{Acute Studies-Outcomes}

The most relevant findings are discussed according to outcomes in the following subsections (also see Table 3).

Hydration. Regarding preexercise consumption, Castro-Sepulveda et al. (2016) found no between-treatment differences (percentage change from predrink to postexercise) or Time $\times$ Trial interactions for body mass, plasma $\mathrm{Na}^{+}$or $\mathrm{K}^{+}$, or urine specific gravity when $700 \mathrm{ml}$ of $4.6 \%$ beer, $0 \%$ beer, or water was consumed 45 min preexercise. There were also no differences in sweat rate.

Postexercise beer consumption affected hydration-related outcomes to varying degrees in all relevant studies (Desbrow et al., 2013, 2015, 2019; Flores-Salamanca \& Aragón-Vargas, 2014; Hobson \& Maughan, 2010; Shirreffs \& Maughan, 1997; Wijnen et al., 2016) except Jiménez-Pavón et al. (2015). Jiménez-Pavón et al. (2015) reported a lack of between-condition differences in fluid balance, urine volume, urine solutes, hematological/serum parameters, and body composition when they examined ad libitum intake of $4.5 \%$ beer (up to $660 \mathrm{ml}$ followed by water) or mineral water during a 2-hr postexercise period.

Hobson and Maughan (2010) found that, when participants consumed $0 \%$ or $4 \%$ beer in a euhydrated state in the morning after evening cycling, urine volume over $4 \mathrm{hr}$ postconsumption was higher with $4 \%$ beer than $0 \%$ beer. However, no differences existed between $4 \%$ beer and $0 \%$ beer trials when participants were dehydrated. Also, at 1 and $2 \mathrm{hr}$ postconsumption, serum osmolality was greater in both $4 \%$ beer trials than their hydration-matched $0 \%$ beer trials.

Shirreffs and Maughan (1997) evaluated how rehydration was impacted by postexercise beer consumption (equal to $150 \%$ of body mass losses) with differing alcohol contents $(0 \%, 1 \%, 2 \%$, and $4 \%$ ). At $1 \mathrm{hr}$ postrehydration, increases in blood and plasma volumes from prehydration were less with $4 \%$ beer than $0 \%$ beer, but at no other time were between-trial differences statistically significant. Although urine outputs over the 6-hr postrehydration period were not statistically different $(p=.307)$, the median values $(942,1108,1184$, and $1457 \mathrm{ml})$ for $0 \%, 1 \%, 2 \%$, and $4 \%$ beer conditions suggest a possible effect of alcohol content. The small sample $(n=6)$ likely undercut their ability to detect moderate-size differences.

Flores-Salamanca and Aragón-Vargas (2014) utilized a design similar to Shirreffs and Maughan's (1997), but they recruited 11 participants. On three occasions, the participants received water, $0.5 \%$ beer, or $4.6 \%$ beer after they had lost $2.1 \%$ of body mass. The volume administered (100\% of body mass loss), however, was lower than Shirreffs and Maughan's (1997). Overall, the fluid retention was lower and urine output was higher over a 3-hr postconsumption period for $4.6 \%$ beer than other conditions.

Like Flores-Salamanca and Aragón-Vargas (2014), Wijnen et al. (2016) had participants replace exercise-induced fluid losses equaling $100 \%$ of body mass loss. However, they had five conditions ( $0 \%$ beer, $2 \%$ beer, $5 \%$ beer, sports drink, and water) versus three. The $5 \%$ beer increased urine output and worsened fluid balance versus sports drink at $1 \mathrm{hr}$ posthydration, but these differences were not sustained $2-5 \mathrm{hr}$ postconsumption. Notably, Wijnen et al. (2016) induced mild dehydration (1\% body mass); consequently, fluid and alcohol consumed for the beer conditions were less than that consumed for Flores-Salamanca and AragónVargas (2014).

Desbrow et al. (2019) had undergraduates consume $0.9 \%$ beer or Gatorade ad libitum after running $10 \mathrm{~km}$. Gatorade was consumed in larger quantities than $0.9 \%$ beer $(1.05$ vs. $0.85 \mathrm{~L})$ over 30-60 min and was rated higher for liking and refreshment. Furthermore, water intake over the rest of the day was higher with Gatorade than beer (3.34 vs. $2.98 \mathrm{~L}$ ).

Two additional studies from Desbrow et al. (2013, 2015) examined interactions between the alcohol and $\mathrm{Na}^{+}$contents of beer. Their 2013 investigation found that, when $25 \mathrm{mmol} / \mathrm{L} \mathrm{Na}^{+}$is added to $2.3 \%$ beer, it enhances postexercise rehydration (lower urine volume and better net fluid balance) relative to $4.8 \%$ beer, even if $4.8 \%$ beer contains $25 \mathrm{mmol} / \mathrm{L} \mathrm{Na}^{+}$. Desbrow et al. (2015) extended these findings by using a midstrength beer (3.5\%), as well as by adding more $\mathrm{Na}^{+}$to low-alcohol beer. Urine volume over $5 \mathrm{hr}$ 


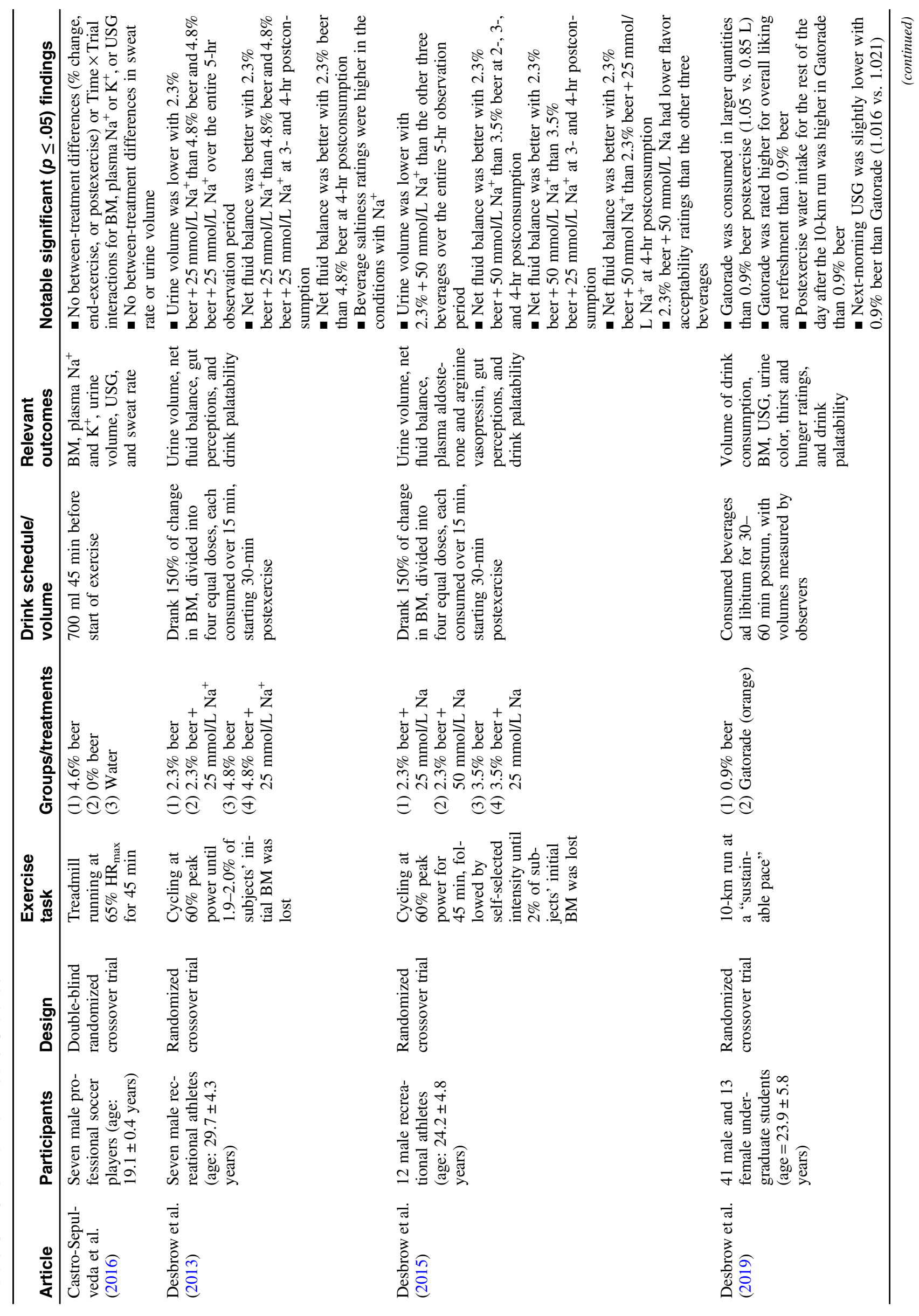

IJSNEM Vol. 31, No. 5, 2021 


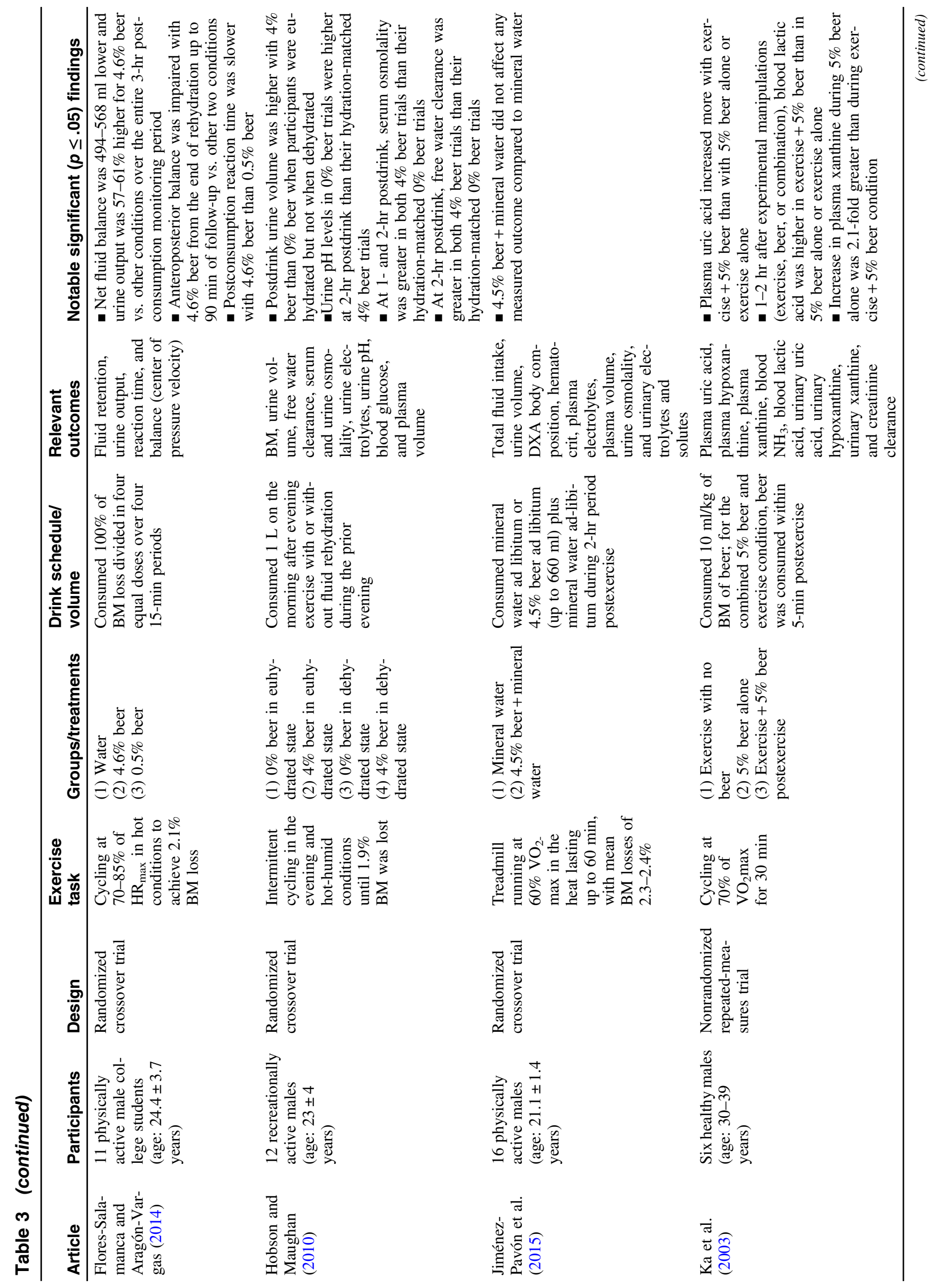




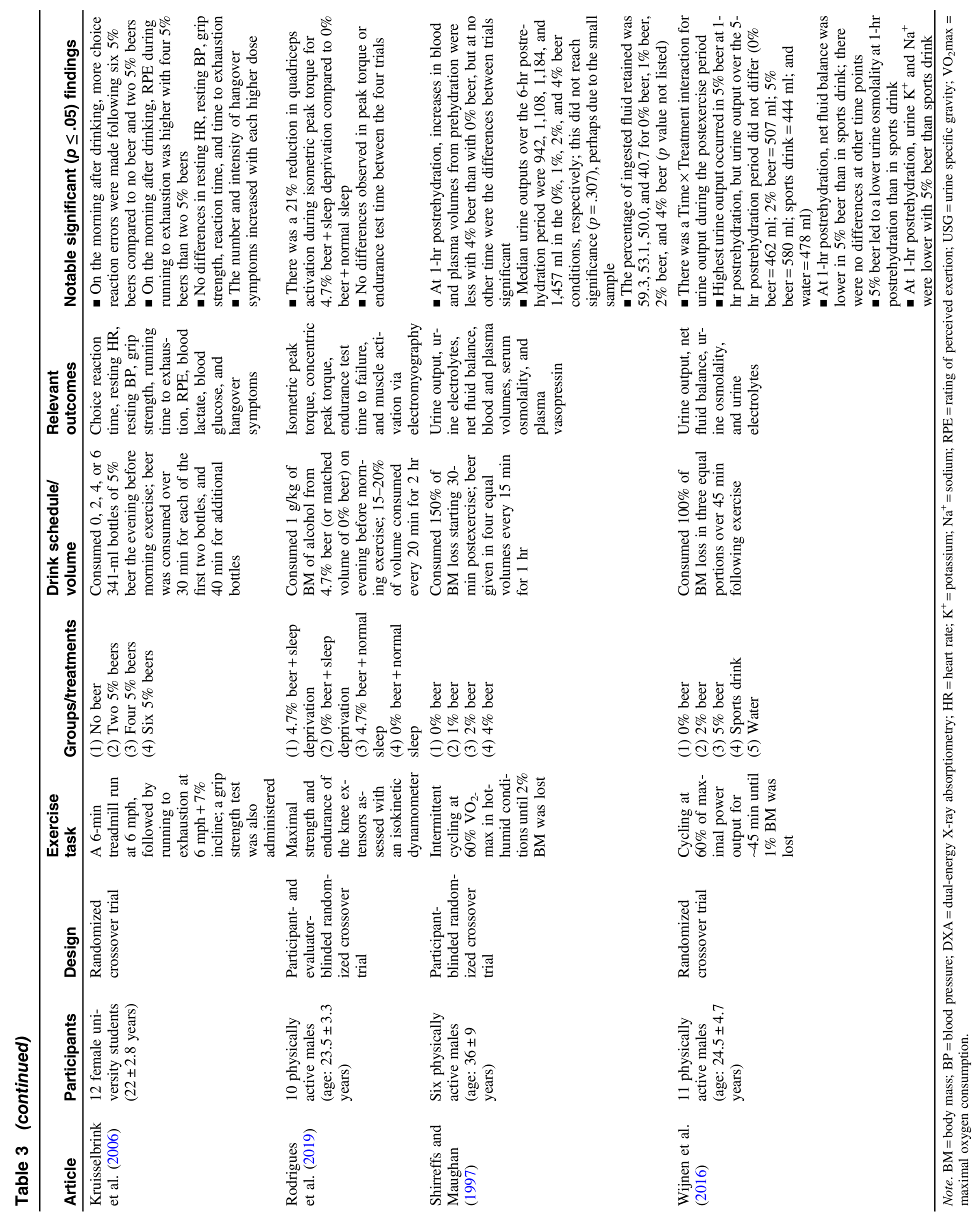


was lower with $2.3 \%$ beer that had $50 \mathrm{mmol} / \mathrm{L} \mathrm{Na}^{+}$versus $2.3 \%$ beer with $25 \mathrm{mmol} / \mathrm{L} \mathrm{Na}^{+}, 3.5 \%$ beer alone, or $3.5 \%$ beer with $25 \mathrm{mmol} / \mathrm{L} \mathrm{Na}^{+}$. Additionally, fluid balance was better at several time points with the $2.3 \%$ beer that had $50 \mathrm{mmol} / \mathrm{L} \mathrm{Na}^{+}$. Across both studies, the participants rated flavor as lower or drinks as saltier when $\mathrm{Na}^{+}$was added.

Neuromuscular performance. Two studies looked at the "hangover" effects of beer. The effects of $4.7 \%$ and $0 \%$ beers with or without sleep deprivation were evaluated by Rodrigues et al. (2019). In comparison to $0 \%$ beer combined with normal sleep, a significant $21 \%$ reduction in isometric quadriceps activation was observed with $4.7 \%$ beer and sleep deprivation. But no differences in performance (isometric peak torque, concentric peak torque, and endurance time to failure) were observed. Similarly, Kruisselbrink et al. (2006) reported that grip strength was not statistically different when the participants consumed zero, two, four, or six bottles of $5 \%$ beer the evening before the morning testing.

Beyond looking at the effects of water, low-alcohol beer $(0.5 \%)$, and $4.6 \%$ beer on rehydration, Flores-Salamanca and Aragón-Vargas (2014) examined balance while each participant stood for $30 \mathrm{~s}$ on their left foot. Anteroposterior balance was impaired with $4.6 \%$ beer for 90 min postconsumption relative to other beverages.

Miscellaneous outcomes. Ka et al. (2003) looked at plasma uric acid following $30 \mathrm{~min}$ of cycling and whether ingesting $5 \%$ beer postexercise modified responses. Uric acid levels were highest following exercise plus 5\% beer when compared to consuming 5\% beer alone or exercise alone. When blood lactate was measured $1-2 \mathrm{hr}$ postexercise, the levels were higher in the $5 \%$ beer-exercise condition than with beer alone or exercise alone.

Kruisselbrink et al. (2006) examined choice reaction time, as well as cardiovascular responses, blood markers, and rating of perceived exertion during submaximal and maximal running in females on the morning after the evening consumption of zero, two, four, and six bottles of $5 \%$ beer. Time to exhaustion was measured during maximal running. When subjects consumed four bottles, the rating of perceived exertion was higher compared with two bottles (17.9 vs. 17.1). For choice reaction time, subjects made more errors following the consumption of six bottles versus two and zero bottles (1.92 vs. 0.83 and 0.42 . respectively).

Flores-Salamanca and Aragón-Vargas (2014) induced dehydration through cycling in hot conditions and tested reaction time after beverage rehydration by asking subjects to press a key as quickly as possible when an image was presented on a screen. The reaction time for $4.6 \%$ beer was longer $(0.314 \pm 0.036 \mathrm{~s})$ than for $0.9 \%$ beer $(0.294 \pm 0.034 \mathrm{~s})$, though the difference with water wasn't significant $(0.293 \pm 0.049 \mathrm{~s}, p=.077)$.

\section{Chronic Studies-Samples}

Four studies used chronic protocols (Table 4). Two of these reported different outcomes from a 10-week experiment that recruited men and women who were previously untrained (Molina-Hidalgo et al., 2019; Molina-Hidalgo et al., 2020). Scherr et al. (2012) enrolled runners training for a marathon, while the fourth study recruited sedentary men (Cox et al., 1993). In terms of sex, the Molina-Hidalgo et al. $(2019,2020)$ studies reported an almost identical proportion of males and females ( $\sim 52 \%$ vs. $48 \%$ ), with the remaining studies consisting of men. The mean/median ages ranged from 24 to 44 years. The sample sizes varied from 72 to 121 participants.

\section{Chronic Studies-Exercise Components}

The exercise components varied and included cycling (Cox et al., 1993), running (Scherr et al., 2012), and HIIT (Molina-Hidalgo et al., 2019; Molina-Hidalgo et al., 2020). The frequency of exercise ranged from two to three times/week (Cox et al., 1993; Molina-Hidalgo et al., 2019; Molina-Hidalgo et al., 2020), with one exception being Scherr et al. (2012), who did not incorporate a new exercise intervention, but enrolled runners preparing for the $\mathrm{Mu}-$ nich Marathon. In terms of intensity, two involved HIIT (MolinaHidalgo et al., 2019; Molina-Hidalgo et al., 2020), one study prescribed continuous exercise at low and moderate loads (Cox et al., 1993), and one allowed participants to self-select the intensity (Scherr et al., 2012).

\section{Chronic Studies-Beer Consumption Procedures}

One study looked at differences between low- and normalalcoholic beer and provided 750-ml bottles that were consumed ad libitum over 4 weeks (Cox et al., 1993). Two articles reported results from a study that compared moderate intakes of normal beer, nonalcoholic beer, vodka, or sparkling water for 10 weeks (Molina-Hidalgo et al., 2019; Molina-Hidalgo et al., 2020). Finally, Scherr et al. (2012) compared consuming 1.0-1.5 L of nonalcoholic, polyphenol-rich beer to a low-polyphenol placebo over 5 weeks.

\section{Chronic Studies-Outcomes}

The outcomes had little overlap (Table 4); hence, each study is presented as a separate subsection.

Cox et al. (1993). Cox et al. (1993) used a two-way factorial randomized trial to evaluate whether the amount of alcohol consumed (six $750-\mathrm{ml}$ bottles/week of $0.9 \%$ or $5.0 \%$ beer) modified adaptations to training (vigorous or light) in habitual moderate-toheavy drinkers. Over 4 weeks, $\mathrm{VO}_{2} \max$ increased by $10 \%$ with vigorous training, irrespective of the type of beer ingested. Systolic and diastolic blood pressures declined in the supine and standing positions (by roughly $2-5 \mathrm{mmHg}$ ) with $0.9 \%$ beer versus $5.0 \%$ beer. The groups receiving $0.9 \%$ beer also had decreases in serum triglycerides, high-density lipoprotein (HDL), apo A-I, and apo A-II, with an increase in total-to-HDL cholesterol ratio. A main effect of $0.9 \%$ beer was seen to reduce body mass by $0.43 \mathrm{~kg}$, and there was a corresponding main effect of vigorous exercise on body mass $(-0.43 \mathrm{~kg})$. There did not appear to be interactions between beer type and exercise intensity.

Molina-Hidalgo et al. (2019, 2020). Two articles reported different outcomes from the same 10-week intervention, involving varying combinations of HIIT and alcohol intake. There were both randomized and nonrandomized aspects to the allocation. The participants first chose whether they were to be allocated to HIIT or no exercise. The participants who chose HIIT then chose whether to consume alcohol or not. Finally, the participants who chose alcohol were randomized to either $5.4 \%$ beer or a sparkling water-vodka mix, while those who chose no alcohol were randomized to $0 \%$ beer or sparkling water.

The 2020 article reported that $\mathrm{VO}_{2}$ max and exhaustion time increased in all four HIIT-beverage groups, with no change in the no-HIIT-no-beverage group. There did not appear to be pairwise differences in $\mathrm{VO}_{2}$ max changes between the four HIIT-beverage groups. When assessing neuromuscular outcomes, there were no group effects or Group $\times$ Time interactions for handgrip strength or 


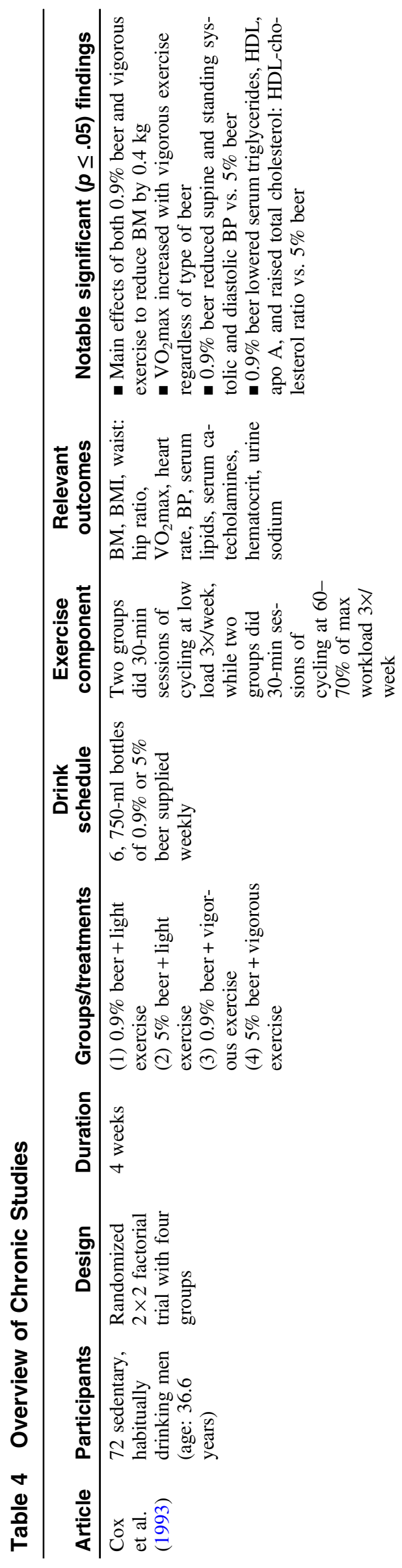

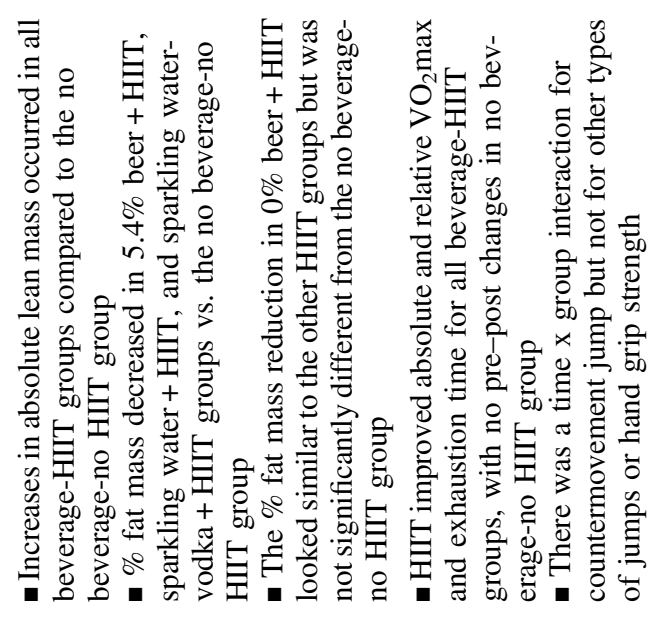

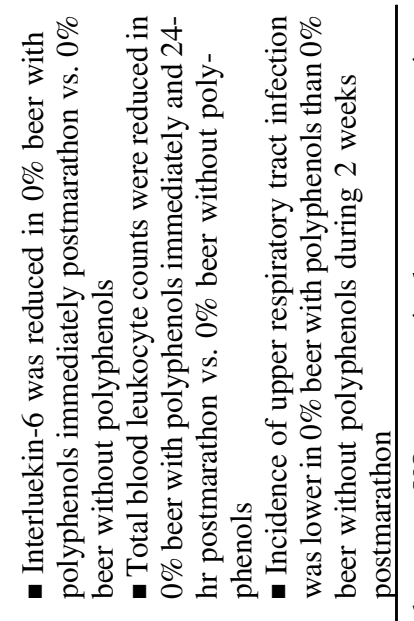

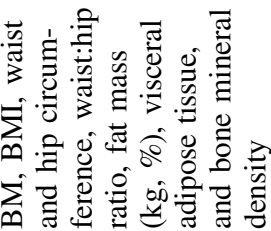
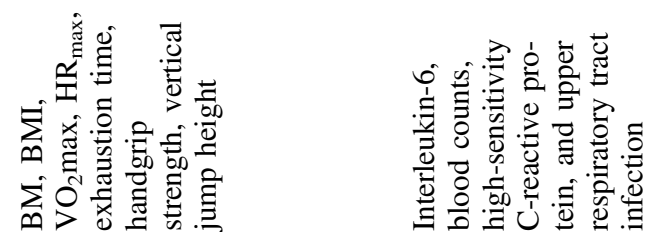

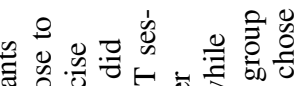

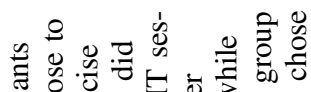

䕇密声

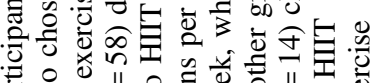

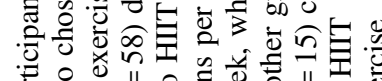

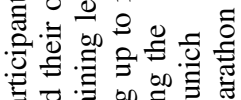

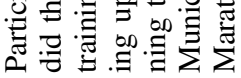

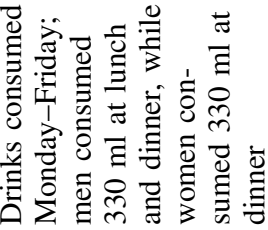

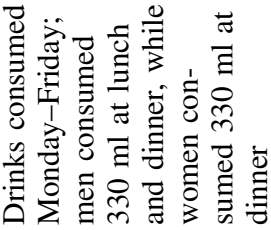

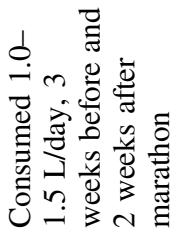

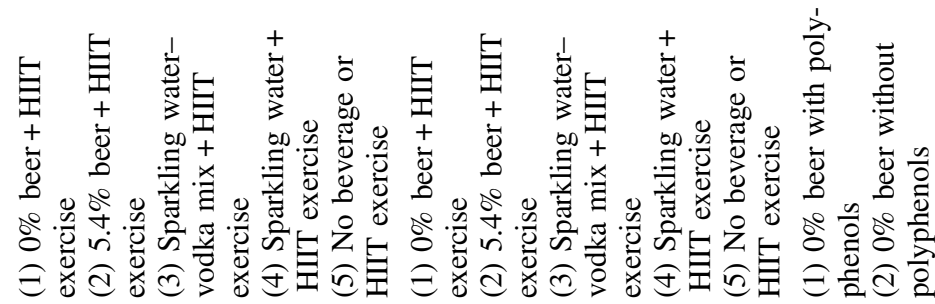

$\frac{n}{8}$
$\frac{8}{3}$
0
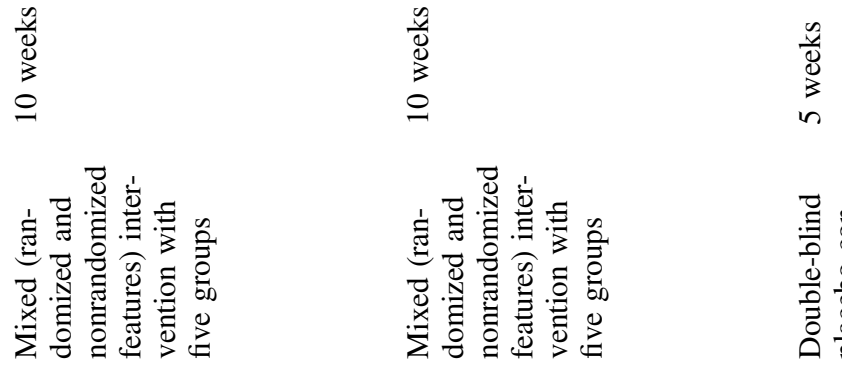

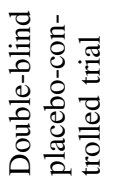

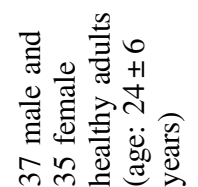

芯苇

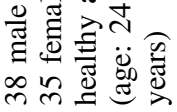

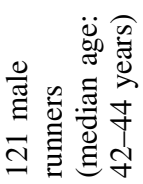

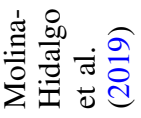

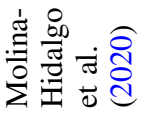

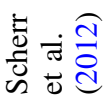


jump heights, except for the countermovement jump, which showed a Time $\times$ Group interaction. Molina-Hidalgo et al. (2019) found similar increases in lean mass and decreases in fat mass in all four HIIT-beverage groups versus the no-HIIT-no-beverage group.

Scherr et al. (2012). For 3 weeks before and 2 weeks after the Munich Marathon, participants consumed 1.0-1.5 L/day of 0\% beer with or without polyphenols. Immediately postmarathon, interluekin-6 and blood leukocyte counts were reduced in the polyphenol-rich beer group. Leukocyte counts at $24 \mathrm{hr}$ postrace were also lower with polyphenol-rich beer. For 2 weeks postmarathon, the low-polyphenol beer group had 3.25 times the odds of experiencing an upper respiratory tract infection relative to the polyphenol-rich group.

\section{Discussion}

\section{Acute Studies}

When looking at acute responses to beer intake, there were mixed results, which is relatively unsurprising, given the variation in outcomes, types of beer consumed, and exercise tasks used. Of note, numerous studies from our review focused on hydrationrelated outcomes. Based on postexercise rehydration studies, choosing lower alcohol beer and/or adding $\mathrm{Na}^{+}$may aid with fluid retention, at least when fluid intake is standardized to body mass. Most studies reviewed, however, did not allow for an ad libitum rehydration strategy nor the consumption of food postexercise. When individuals are given the option to consume beverages with food ad libitum, the type of beverage may have less of an impact on fluid restoration (Campagnolo et al., 2017), whereas studies that standardize drink volume and do not provide access to food show that beverage composition considerably impacts fluid recovery (Hobson \& Maughan, 2010; Shirreffs et al., 2007). As such, studies that do not consider ad libitum drinking and eating may give a misleading picture of a beverage's effects in real-world contexts. Ultimately, given that only two studies identified in this review used ad libitum drinking postexercise (Desbrow et al., 2019; Jiménez-Pavón et al., 2015), it is difficult to extrapolate the results to naturalistic settings in which people have more control over the volume of beer ingested as well as the coconsumption of food.

With this caveat in mind, several studies showed that, when normal beer $(>4 \%$ alcohol) is consumed in standardized amounts postexercise, fluid balance and hydration are modestly worsened. Practically, this would be most problematic when an athlete has a brief recovery between exercise bouts, whereas the implications are unclear when there is $>6-8 \mathrm{hr}$ of recovery. Regardless, it is inadvisable to use normal alcoholic beer as the sole rehydration source when a fluid deficit of $\geq 1.5 \%$ of body mass is created. For example, a person weighing $75 \mathrm{~kg}$ would need to consume $1.13 \mathrm{~L}$ of beer to fully recover a body mass loss of $1.5 \%$. The larger the dose of alcohol, the larger the diuretic effect (Jones, 1990). For individuals who wish to prioritize rehydration while consuming normal- or high-alcohol beer postexercise, they should consider drinking a limited amount $(<700 \mathrm{ml})$ while focusing on coingestion of water, sports drinks, or other suitable rehydration beverages (e.g., milk). As shown by Jiménez-Pavón et al. (2015), mixing moderate beer intake $(660 \mathrm{ml})$ with water may be just as effective as consuming water alone.

A variety of other factors that could impact the volume and type of beer ingested on any given occasion were not accounted for in the studies identified. First, one major consideration is that drinking guidelines and norms vary between countries and are influenced by national culture (Silva et al., 2017). Annual per capita beer consumption in Ireland in 2005, for example, was over 160 and $<40 \mathrm{~L}$ in Italy, France, and China (Colen \& Swinnen, 2011). Second, negative emotion (stress, anger, and depression) and poor emotional regulation strategies predict alcohol craving (Kim \& Kwon, 2020). Similarly, positive interpersonal experiences can influence individuals to drink more socially (Mohr et al., 2001). These findings suggest that people are inclined to drink to cope with both positive and negative emotions. Practically speaking, this means that a person's emotional state in the postexercise period may impact the volume of beer ingested. Besides psychological and sociocultural factors, one must consider biological, intrinsic attributes, extrinsic sensory characteristics, and situational factors as well (Betancur et al., 2020). Virtually none of the studies identified in this review examined how these factors mediate beer consumption.

When it comes to fluid ingestion, there is a balance between what is considered palatable and physiologically recommendable. Desbrow et al. $(2013,2015)$ observed decreases in palatability or increased saltiness with added $\mathrm{Na}^{+}$. To what extent does one make concessions in taste for the sake of recovery when that drink is consumed for pleasure? It is hypothetically possible that, under ad libitum conditions, people will consume less beer if it has substantial amounts of $\mathrm{Na}^{+}$, which would undercut the benefit of the added sodium with respect to fluid balance. Consequently, future studies should evaluate the ad libitum consumption of $\mathrm{Na}^{+}$-enriched beer postexercise.

The only study to have participants consume beer preexercise revealed no differences in body mass, plasma $\mathrm{Na}^{+}$or $\mathrm{K}^{+}$, urine specific gravity, or sweat rate (Castro-Sepulveda et al., 2016). Alcohol consumption, even in moderate amounts, can negatively impact neuromotor functioning (Demura \& Uchiyama, 2008). Consequently, preexercise alcoholic beer consumption is uncommon. Although alcoholic beer contains carbohydrate and fluid, the amount that would need to be consumed to meet standard preexercise recommendations for the intakes of these nutrients would be prohibitive. Whether nonalcoholic beer can serve as a suitable preexercise beverage has not been thoroughly studied. Nonalcoholic beer typically contains less carbohydrate by volume than sports drinks, and significantly less sodium.

For neuromuscular effects, alcoholic beer can be detrimental in several ways, at least when more than 1 liter is consumed. Balance is affected for up to 90 min postingestion (Flores-Salamanca \& AragónVargas, 2014), and reaction time is also impaired in the hours after consumption (Flores-Salamanca \& Aragón-Vargas, 2014). In terms of next-day "hangover" effects, choice reaction time may be negatively affected with large doses of alcoholic beer (Kruisselbrink et al., 2006), and muscle activation could be negatively impacted when beer is mixed with sleep restriction (Rodrigues et al., 2019). In contrast, alcoholic beer does not seem to impact muscle strength or endurance when consumed the night before testing.

\section{Chronic Studies}

The reduction in upper respiratory tract infections in Scherr et al. (2012) suggests that polyphenols found in beer may exert antiviral and inflammation-reducing properties. This finding has implications, in that a $0 \%$ beer may be efficacious in preventing respiratory infections during heavy training or following strenuous competition. Whether these immune effects extend to alcoholic beer is undetermined, but the amount of nonalcoholic beer (1.0-1.5 L/day) used by Scherr et al. (2012) could be prohibitive if applied to beer with a normal or high alcohol content. 
Moderate beer consumption seems to minimally impact cardiorespiratory- and strength-training adaptations, at least at the dosages studied. Molina-Hidalgo et al. (2020) found that a 10 -week HIIT program improved muscular strength via handgrip testing, with no impairment among the $5.4 \%$ beer group. Similarly, $\mathrm{VO}_{2} \mathrm{max}$ and treadmill time to exhaustion increased with HIIT, regardless of the type of beverage. This finding is in line with Cox et al. (1993), who found increases in $\mathrm{VO}_{2} \mathrm{max}$ with vigorous exercise, regardless of whether normal- or low-alcohol beer was ingested. Still, caution is warranted in extrapolating these results to elite athletes or other types of training until more research is completed.

There is still ambiguity as to how beer intake influences body composition. Molina-Hidalgo et al. (2019) showed that moderate beer consumption (either from $5.4 \%$ or $0 \%$ beer) combined with HIIT did not lead to notable differences in body mass, body fat, or lean mass changes relative to HIIT with water or vodka. In contrast, Cox et al. (1993) found a small reduction in body mass when subjects switched from drinking normal to low-alcohol beer for 4 weeks. However, it is difficult to determine to what extent the small reduction in mass $(-0.43 \mathrm{~kg})$ would continue to accumulate over time. It is also essential to comment that beer's effects on body composition/weight may depend on the doses of exercise and beer. High daily dosages of alcoholic beer could impair lean mass accretion, given that binge drinking acutely interferes with postexercise MPS (Parr et al., 2014) and that studies of chronic alcoholism show impaired MPS (Pacy et al., 1991). Furthermore, heavy beer drinking adds substantial energy to the diet, potentially resulting in body fat accumulation. Individuals doing large amounts of exercise may be somewhat protected from these effects, but this suggestion requires further study. Given the small number of studies on beer intake and weight/body composition in the setting of exercise training, it is premature to make strong conclusions. For now, it appears as if consuming 1-2 beers/day has negligible effects on body composition/weight.

Finally, Cox et al. (1993) examined changes to cardiovascular risk factors with alcoholic beer restriction and exercise among previously sedentary men. Switching to low-alcohol beer modestly reduced resting blood pressure, serum triglycerides, HDL cholesterol, and apolipoprotein A subfractions, regardless of the type of exercise (light vs. vigorous). It should be noted that participants were relatively heavy drinkers at the baseline (approximately 470$480 \mathrm{ml}$ of ethanol/week), and intake was reduced by $85 \%$ with lowalcohol beer. Whether a modest change in intake would alter cardiovascular biomarkers is debatable, but some experimental evidence suggests it would (Chiva-Blanch et al., 2015).

\section{Limitations}

Most sample sizes were small, limiting the statistical power to detect small differences between conditions. Furthermore, most studies utilized continuous steady-state exercise to study the pre- or postexercise effects of beer ingestion on hydration. Future studies should incorporate exercise with varying intensities, durations, and types of exercise to increase ecological validity. It is difficult to blind subjects to alcoholic beverages, if not impossible, making certain outcomes more prone to bias. Dehydration was limited to various percentages of body weight, fluid replacement varied from $100 \%$ to $150 \%$ of body mass loss postexercise, and few studies used ad libitum drinking. Most of the research focused on men, and beer ingestion could differentially affect outcomes in women due to body size/composition, sweat rates, speed of alcohol metabolism, and so on. Finally, chronic studies lasted from 4 to 10 weeks, not an ideal amount of time when seeking to understand how beer affects body composition or fitness changes.

\section{Conclusion}

In general, low-alcohol beer seems to be more efficacious when individuals are looking to quickly rehydrate postexercise. Adding $\mathrm{Na}^{+}$to alcoholic beer may improve its rehydration properties, but there may be tradeoffs related to palatability. These conclusions are largely based on studies that standardized beverage volume, and the results may not apply to situations where people ingest fluids and food in an ad libitum fashion. When choosing a beer higher in alcoholic content postexercise $(>4 \%)$, it is wise to pair it with nonalcoholic rehydration options to mitigate alcohol-induced diuresis, particularly when exercise-induced fluid losses are large. Nonalcoholic, polyphenol-rich beer has been observed to provide respiratory protection following a marathon, suggesting it could be a strategy for preventing respiratory infections during heavy training. Chronic changes in body composition, as well as muscle performance, adaptation, and recovery, seem largely unaffected by moderate beer consumption.

\section{Acknowledgments}

The literature review protocol was developed by P.B. Wilson. The literature search and study appraisal were conducted by J.L. Wynne and P.B. Wilson. Writing, editing, and preparation of the manuscript were performed by P.B. Wilson and J.L. Wynne. Both authors approved the final version of the manuscript.

\section{References}

Babor, T.F., Xuan, Z., Damon, D., \& Noel, J. (2013). An empirical evaluation of the US Beer Institute's self-regulation code governing the content of beer advertising. American Journal of Public Health, 103(10), e45-e51. PubMed ID: 23947318 doi:10.2105/AJPH.2013. 301487

Barnes, M.J. (2014) Alcohol: Impact on sports performance and recovery in male athletes. Sports Medicine, 44(7), 909-919. doi:10.1007/ s40279-014-0192-8

Betancur, M.I., Motoki, K., Spence, C., \& Velasco, C. (2020). Factors influencing the choice of beer: A review. Food Research International, 137, 109367. PubMed ID: 33233069 doi:10.1016/j.foodres. 2020.109367

Campagnolo, N., Iudakhina, E., Irwin, C., Schubert, M., Cox, G.R., Leveritt, M., \& Desbrow, B. (2017). Fluid, energy and nutrient recovery via ad libitum intake of different fluids and food. Physiology \& Behavior, 171, 228-235. PubMed ID: 28104353 doi:10.1016/j. physbeh.2017.01.009

Castro-Sepulveda, M., Johannsen, N., Astudillo, S., Jorquera, C., Álvarez, C., Zbinden-Foncea, H., \& Ramírez-Campillo, R. (2016). Effects of beer, non-alcoholic beer and water consumption before exercise on fluid and electrolyte homeostasis in athletes. Nutrients, 8(6), 345. doi:10.3390/nu8060345

Chiva-Blanch, G., Magraner, E., Condines, X., Valderas-Martínez, P., Roth, I., Arranz, S., \& Estruch, R. (2015). Effects of alcohol and polyphenols from beer on atherosclerotic biomarkers in high cardiovascular risk men: A randomized feeding trial. Nutrition, Metabolism and Cardiovascular Diseases, 25(1), 36-45. PubMed ID: 25183453 doi:10.1016/j.numecd.2014.07.008 
Colen, L.\& Swinnen, J.F.M. (2011). Beer drinking nations: The determinants of global beer consumption. In Johan F.M. Swinnen (Ed.), The economics of beer (pp. 123-140). Oxford: Oxford University Press.

Cox, K.L., Puddey, I.B., Morton, A.R., Beilin, L.J., Vandongen, R., \& Masarei, J.R. (1993). The combined effects aerobic exercise and alcohol restriction on blood pressure and serum lipids: A two way factorial study in sedentary men. Journal of Hypertension, 11(2), 191-201. doi:10.1097/00004872-199302000-00012.

de Morton N.A. (2009). The PEDro scale is a valid measure of the methodological quality of clinical trials: A demographic study. Australian Journal of Physiotherapy, 55(2), 129-133. doi:10.1016/ S0004-9514(09)70043-1

Demura, S., \& Uchiyama, M. (2008). Influence of moderate alcohol ingestion on gait. Sport Sciences for Health, 4(1-2), 21-26. doi:10. 1007/s11332-008-0062-6

Desbrow, B., Barnes, K., Cox, G.R., Iudakhina, E., McCartney, D., Skepper, S., Young, C., \& Irwin, C. (2019). Calorie-containing recovery drinks increase recreational runners' voluntary energy and carbohydrate intake, with minimal impact on fluid recovery. International Journal of Sport Nutrition and Exercise Metabolism, 29(2), 220-227. PubMed ID: 31141394 doi:10.1123/ijsnem.20180269

Desbrow, B., Cecchin, D., Jones, A., Grant, G., Irwin, C., \& Leveritt, M. (2015). Manipulations to the alcohol and sodium content of beer for postexercise rehydration. International Journal of Sport Nutrition and Exercise Metabolism, 25(3), 262-270. PubMed ID: 25588064 doi:10.1123/ijsnem.2014-0064

Desbrow, B., Murray, D., \& Leveritt, M. (2013). Beer as a sports drink? Manipulating beer's ingredients to replace lost fluid. International Journal of Sport Nutrition and Exercise Metabolism, 23(6), 593-600. PubMed ID: 23690556 doi:10.1123/ijsnem.23.6.593

Flores-Salamanca, R., \& Aragón-Vargas, L. F. (2014). Postexercise rehydration with beer impairs fluid retention, reaction time, and balance. Applied Physiology, Nutrition, and Metabolism, 39(10), 1175-1181. PubMed ID: 25041559 doi:10.1139/apnm-2013-0576

Gallup. (2019) Alcohol and drinking. Retrieved from https://news.gallup. com/poll/1582/alcohol-drinking.aspx

Ghiselli, A., Natella, F., Guidi, A., Montanari, L., Fantozzi, P., \& Scaccini, C. (2000). Beer increases plasma antioxidant capacity in humans. Journal of Nutritional Biochemistry, 11(2), 76-80. doi:10.1016/ S0955-2863(99)00077-7

Hobson, R.M., \& Maughan, R.J. (2010). Hydration status and the diuretic action of a small dose of alcohol. Alcohol and Alcoholism, 45(4), 366-373. PubMed ID: 20497950 doi:10.1093/alcalc/agq029

Jiménez-Pavón, D., Cervantes-Borunda, M.S., Díaz, L.E., Marcos, A., \& Castillo, M.J. (2015). Effects of a moderate intake of beer on markers of hydration after exercise in the heat: A crossover study. Journal of the International Society of Sports Nutrition, 12(1), 26. PubMed ID: 26056515 doi:10.1186/s12970-015-0088-5

Jones, A.W. (1990). Excretion of alcohol in urine and diuresis in healthy men in relation to their age, the dose administered and the time after drinking. Forensic Science International, 45(3), 217-224. PubMed ID: 2361644 doi:10.1016/0379-0738(90)90177-Z

Ka, T., Yamamoto, T., Moriwaki, Y., Kaya, M., Tsujita, J., Takahashi, S., . . . Hada, T. (2003). Effect of exercise and beer on the plasma concentration and urinary excretion of purine bases. Journal of Rheumatology, 30, 1036-1042.

Kim, S., \& Kwon, J.H. (2020). The impact of negative emotions on drinking among individuals with social anxiety disorder in daily life: The moderating effect of maladaptive emotion regulation strategies. Cognitive Therapy and Research, 44(2), 345-359. doi:10.1007/ s10608-019-10045-8
Kontro, T.K., Sarna, S., Kaprio, J., \& Kujala, U.M. (2017). Use of alcohol and alcohol-related morbidity in Finnish former elite athletes. Medicine and Science in Sports and Exercise, 49(3), 492-499. PubMed ID: 27787336 doi:10.1249/MSS.0000000000001137

Kruisselbrink, L.D., Martin, K.L., Megeney, M., Fowles, J.R., \& Murphy, R.J. (2006). Physical and psychomotor functioning of females the morning after consuming low to moderate quantities of beer. Journal of Studies on Alcohol, 67(3), 416-420. PubMed ID: 16608151 doi:10. 15288/jsa.2006.67.416

Leasure, J.L., Neighbors, C., Henderson, C.E., \& Young, C.M. (2015). Exercise and alcohol consumption: What we know, what we need to know, and why it is important. Frontiers in Psychiatry, 6, 156. doi:10. 3389/fpsyt.2015.00156

Logan, B.K., Case, G.A., \& Distefano, S. (1999). Alcohol content of beer and malt beverages: Forensic considerations. Journal of Forensic Science, 44(6), 14603J-1295. doi:10.1520/JFS14603J

Martin, M. (1998). The use of alcohol among NCAA division I female college basketball, softball, and volleyball athletes. Journal of Athletic Training, 33(2), 163-167. PubMed ID: 16558505

Mohr, C.D., Armeli, S., Tennen, H., Carney, M.A., Affleck, G., \& Hromi, A. (2001). Daily interpersonal experiences, context, and alcohol consumption: Crying in your beer and toasting good times. Journal of Personality and Social Psychology, 80(3), 489-500. PubMed ID: 11300581 doi:10.1037/0022-3514.80.3.489

Molina-Hidalgo, C., De-la-O, A., Dote-Montero, M., Amaro-Gahete, F.J., \& Castillo, M.J. (2020). Influence of daily beer or ethanol consumption on physical fitness in response to a high-intensity interval training program. The BEER-HIIT study. Journal of the International Society of Sports Nutrition, 17(1), 29. PubMed ID: 32460793 doi:10.1186/ s12970-020-00356-7

Molina-Hidalgo, C., De-la-O, A., Jurado-Fasoli, L., Amaro-Gahete, F.J., \& Castillo, M.J. (2019). Beer or ethanol effects on the body composition response to high-intensity interval training. The BEER-HIIT study. Nutrients, 11(4), 909. doi:10.3390/nu11040909

Moseley, A.M., Herbert, R.D., Maher, C.G., Sherrington, C., \& Elkins, M.R. (2011). Reported quality of randomized controlled trials of physiotherapy interventions has improved over time. Journal of Clinical Epidemiology, 64(6), 594-601. PubMed ID: 21144705 doi:10.1016/j.jclinepi.2010.08.009

Myburgh, K H. (2014). Polyphenol supplementation: Benefits for exercise performance or oxidative stress? Sports Medicine, 44(Suppl. 1), 57-70. doi:10.1007/s40279-014-0151-4

Nelson, T.F., \& Wechsler, H. (2001). Alcohol and college athletes. Medicine and Science in Sports and Exercise, 33(1), 43-47. PubMed ID: 11194110 doi:10.1097/00005768-200101000-00008

O'Brien, K.S., Carr, S., Ferris, J., Room, R., Miller, P., Livingston, M., . . . Lynott, D. (2015). Alcohol advertising in sport and non-sport TV in Australia, during children's viewing times. PloS One, 10(8), e0134889. PubMed ID: 26263170 doi:10.1371/journal.pone.0134889

Overman, S.J., \& Terry, T. (1991). Alcohol use and attitudes: A comparison of college athletes and nonathletes. Journal of Drug Education, 21(2), 107-117. PubMed ID: 1886047 doi:10.2190/KF20-4LER-J9N1-7Q3F

Pacy, P.J., Preedy, V.R., Peters, T.J., Read, M., \& Hallidy, D. (1991). The effect of chronic alcohol ingestion on whole body and muscle protein synthesis-A stable isotope study. Alcohol and Alcoholism, 26(5-6), 505-513. PubMed ID: 1804130 doi:10.1093/oxfordjournals.alcalc. a045152

Parr, E.B., Camera, D.M., Areta, J.L., Burke, L.M., Phillips, S.M., Hawley, J.A., \& Coffey, V.G. (2014). Alcohol ingestion impairs maximal post-exercise rates of myofibrillar protein synthesis following a single bout of concurrent training. PLoS One, 9(2), e88384. PubMed ID: 24533082 doi:10.1371/journal.pone.0088384 
PEDro. (2020) PEDro statistics. Retrieved from https://pedro.org.au/ english/learn/pedro-statistics/\#: :text=The\%20average\%20total\% 20PEDro $\% 20$ score, $10 \% 20$ on $\% 20$ the $\% 20$ PEDro $\% 20$ scale.

Piazza-Gardner, A.K., \& Barry, A.E. (2012). Examining physical activity levels and alcohol consumption: Are people who drink more active? American Journal of Health Promotion, 26(3), e95-e104. doi:10. 4278/ajhp.100929-LIT-328

Piazzon, A., Forte, M., \& Nardini, M. (2010). Characterization of phenolics content and antioxidant activity of different beer types. Journal of Agricultural and Food Chemistry, 58(19), 10677-10683. PubMed ID: 20822144 doi:10.1021/jf101975q

Rodrigues, R., de Azevedo Franke, R., Teixeira, B.C., Macedo, R.C.O., Diefenthaeler, F., Baroni, B.M., \& Vaz, M.A. (2019). Can the combination of acute alcohol intake and one night of sleep deprivation affect neuromuscular performance in healthy male adults? A cross-over randomized controlled trial. Journal of Strength and Conditioning Research, 33(5), 1244-1251. PubMed ID: 30908373 doi:10.1519/JSC.0000000000003124

Scherr, J., Nieman, D.C., Schuster, T., Habermann, J., Rank, M., Braun, S., ... Halle, M. (2012). Nonalcoholic beer reduces inflammation and incidence of respiratory tract illness. Medicine and Science in Sports and Exercise, 44(1), 18-26. PubMed ID: 21659904 doi:10.1249/ MSS.0b013e3182250dda
Shirreffs, S.M., \& Maughan, R.J. (1997). Restoration of fluid balance after exercise-induced dehydration: Effects of alcohol consumption. Journal of Applied Physiology, 83(4), 1152-1158. PubMed ID: 9338423 doi:10.1152/jappl.1997.83.4.1152

Shirreffs, S.M., Watson, P., \& Maughan, R.J. (2007). Milk as an effective post-exercise rehydration drink. British Journal of Nutrition, 98(1), 173-180. doi:10.1017/S0007114507695543

Silva, A.P., Jager, G., Van Zyl, H., Voss, H.P., Pintado, M., Hogg, T., \& De Graaf, C. (2017). Cheers, proost, saúde: Cultural, contextual and psychological factors of wine and beer consumption in Portugal and in the Netherlands. Critical Reviews in Food Science and Nutrition, 57(7), 1340-1349. PubMed ID: 26560863 doi:10.1080/10408398. 2014.969396

Somerville, V., Bringans, C., \& Braakhuis, A. (2017). Polyphenols and performance: A systematic review and meta-analysis. Sports Medicine, 47(8), 1589-1599. PubMed ID: 28097488 doi:10.1007/s40279017-0675-5

Wijnen, A.H., Steennis, J., Catoire, M., Wardenaar, F.C., \& Mensink, M. (2016). Post-exercise rehydration: Effect of consumption of beer with varying alcohol content on fluid balance after mild dehydration. Frontiers in Nutrition, 3, 45. PubMed ID: 27800480 doi:10.3389/fnut.2016.00045

Young, T.W. (n.d.). Beer. Encyclopedia Britannica. Retrieved from https://www.britannica.com/topic/beer 